\title{
DISPEPSIA FUNCIONAL E DEPRESSÃO COMO FATOR ASSOCIADO
}

\author{
Renato Azevedo da SILVA ${ }^{1}$, Ricardo Tavares PINHEIRO², Ricardo Azevedo da SILVA ${ }^{3}$, \\ Bernardo Lessa HORTA ${ }^{4}$, Inácia MORAES 5 e Augusto Duarte FARIA ${ }^{6}$
}

\begin{abstract}
RESUMO - Objetivo - Avaliar a associação entre depressão e dispepsia funcional. Pacientes e métodos - Estudo transversal onde foram avaliados 348 pacientes com diagnóstico de dispepsia no Ambulatório de Gastroenterologia do Hospital Universitário de Pelotas, RS, cidade de médio porte do sul do Brasil, durante o período de 1 ano (de março de 2001 a março de 2002). Após o diagnóstico de dispepsia avaliou-se a presença de depressão, tanto em pacientes com dispepsia funcional, quanto naqueles com dispepsia orgânica. Utilizou-se a análise univariada para descrição das freqüências das variáveis de interesse e da análise bivariada, com o teste qui-quadrado, para comparação entre proporções das variáveis categóricas. A técnica da regressão logística foi utilizada para estabelecer a chance dos pacientes com depressão apresentarem dispepsia funcional e para controlar o efeito das variáveis intervenientes sobre a variável de desfecho. Resultados - Evidenciaram maior prevalência de deprimidos entre os pacientes com dispepsia funcional $(30,4 \%)$ em relação àqueles com dispepsia orgânica $(11,2 \%)$. As mulheres apresentaram maior chance de dispepsia funcional (OR: 1,74, IC 95\%, 1,05-2,89) e, em relação à idade, os intervalos entre 31 e 50 anos (OR: 0,28 IC 95\%, 0,13-0,54) e de 51 a 60 anos (OR: 0,41, IC 95\%, 0,17-0,96) mostraram efeito protetor, ou seja, indivíduos nessas faixas etárias têm menor risco de apresentar dispepsia funcional. Após a análise multivariada, pacientes deprimidos apresentaram chance três vezes maior de co-morbidade com dispepsia funcional do que pacientes não-deprimidos (OR 3,13; IC 95\%; 1,71-5,74).
\end{abstract}

DESCRITORES - Depressão. Dispepsia.

\section{INTRODUÇÃO}

A dispepsia divide-se em dois grandes grupos: um secundário, a doença orgânica, e outro denominado dispepsia funcional (DF). A dispepsia orgânica (DO) pode ser definida como a secundária a lesões específicas, como úlcera péptica, esofagite, câncer gástrico e colelitíase. Assim, é um agrupamento de sintomas resultantes de distintas doenças, sendo estudada a partir das mesmas ${ }^{(21)}$.

No que se refere ao diagnóstico da DF, a falta de uniformidade em relação à definição motivou a realização de reuniões de especialistas de diferentes países, sendo mais conhecidas as de Chicago, nos EUA, e de Cortina e Roma, na Itália. As conclusões do Consenso de Roma II foram publicadas em livro em $2000^{(7)}$ e representam o esforço da comunidade científica para, a partir das investigações realizadas nos últimos anos, sistematizar o conhecimento a respeito dos transtornos gastrointestinais funcionais.
O Consenso de Roma II ${ }^{(32)}$ recomenda que a DF seja diagnosticada quando, em pelo menos 12 semanas consecutivas ou não, nos últimos 12 meses, o paciente apresentar:

1. dor ou desconforto no abdome superior, de caráter persistente ou recurrente, com peso epigástrico pósprandial e/ou saciedade precoce e/ou náuseas e/ou vômitos e/ou distensão abdominal;

2. ausência de doença orgânica que possa explicar os sintomas;

3. ausência de evidências de que a dispepsia é aliviada pela evacuação ou associada à alteração na freqüência ou na forma das fezes.

Essa definição ${ }^{(31)}$ pretende abranger não só os pacientes que apresentam sintomas sem explicação identificável pelos exames, mas também aqueles que apresentam alterações fisiopatológicas ou microbiológicas de relevância clínica incerta para explicar os sintomas como: gastrite por

Estudo realizado no Mestrado em Saúde e Comportamento da Universidade Católica de Pelotas, RS

Endereço para correspondência: Dr. Renato Azevedo da Silva - Mestrado em Saúde e Comportamento - Universidade Católica de Pelotas - UCPel - Rua Almirante Barroso, 1202, prédio G, sala 107 - 96010-280 - Pelotas, RS. E-mail: nuplac@phoenix.ucpel.tche.br 
H. pylori, duodenite, calculose vesicular, hipersensibilidade visceral ou alterações na motilidade gastroduodenal.

O referido Consenso estabeleceu igualmente que pacientes com história clínica de úlcera péptica crônica não devem ser diagnosticados como dispépticos funcionais. Porém, aqueles que tiveram úlcera curada por erradicação do H. pylori e continuam com sintomas devem receber esse diagnóstico. Ainda, segundo o mesmo, as anormalidades fisiológicas mais investigadas em relação à possibilidade de estarem associadas à dispepsia funcional são hipersecreção de ácido gástrico, infecção por H. pylori, dismotilidade gastrointestinal, hipersensibilidade visceral e fatores psicológicos.

DOTEVALL ${ }^{(6)}$ propôs que a DF seja um distúrbio psicossomático, com início multifatorial: fatores psicológicos, sociais e culturais que interagem com variáveis biológicas. A maioria dos estudos sobre o tema ${ }^{(29,30,34)}$ mostra que pacientes com dispepsia apresentam mais interrupções em suas atividades diárias do que indivíduos saudáveis.

A associação entre DF e depressão tem sido avaliada nos últimos anos mediante diferentes métodos e instrumentos de pesquisa. Uma revisão dos principais estudos sobre DF e sintomas psicológicos ${ }^{(4,9,12,16,17,19,24,27)}$ mostra que a prevalência de depressão ou de sintomas depressivos é alta nos dispépticos funcionais, indicando a existência de uma associação.

A bibliografia científica não apresenta estudos brasileiros que abordem o tema e são poucos os estudos estrangeiros que utilizam a entrevista clínica para confirmar o diagnóstico de depressão nos pacientes. Sendo assim, o objetivo deste estudo foi verificar se depressão é fator associado à DF e investigar outros fatores de risco para o problema.

\section{MÉTODOS}

O presente estudo faz parte do protocolo de investigação de um ensaio clínico com o objetivo de estabelecer medidas efetivas de intervenção na DF. Foi aprovado pelo Comitê de Ética local em 1999, e os pacientes, como critério de inclusão, assinaram um termo de consentimento informado, detalhando os procedimentos e objetivos da investigacão. Todos os pacientes que apresentaram diagnóstico positivo para depressão foram encaminhados para atendimento no Ambulatório de Psiquiatria do Hospital Universitário da Universidade Católica de Pelotas, RS.

A avaliação inicial buscou diagnosticar dispepsia e identificar pacientes dispépticos orgânicos e funcionais. As entrevistas foram realizadas entre março de 2001 e março de 2002, no Ambulatório de Gastroenterologia do Hospital Universitário. A amostra selecionada apresentava diagnóstico inicial de dispepsia e idade entre 18 e 65 anos. Nesta mesma etapa foi aplicado um instrumento de avaliacão socioeconômica.

No processo de diagnóstico foram utilizados os instrumentos propostos por Roma II e os seguintes exames complementares: endoscopia digestiva, ultra-sonografia, exames laboratoriais (uréia, creatinina, fosfatase alcalina, tempo de protrombina, bilirrubinas, TGO, TGP, albumina, glicemia, prolactina, LH, FSH, TRH ou TSH, T3 e T4, hemograma e exame parasitológico de fezes). Para identificar a infecção por $H$. pylori foi realizado exame histopatológico. Os pacientes enquadrados nos critérios de inclusão e que apresentaram queixa de cólica biliar, intolerância à gordura e vômito pós-prandial foram encaminhados para o exame ultra-sonográfico do abdome, com a finalidade de controlar a variável presença de cálculo biliar (Figura 1). Os pacientes que não apresentavam alterações nos exames que justificassem os sintomas eram considerados como dispépticos funcionais.

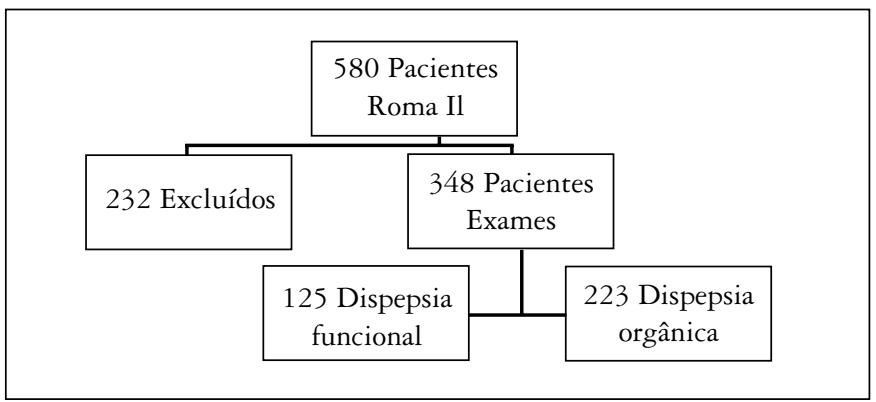

FIGURA 1 - Fluxograma da construção da amostra

A avaliação econômica foi realizada através da renda declarada pelos pacientes e da aplicação de uma escala de avaliação econômica. Essa classificação enquadra as pessoas em classes (A, B, C, D, ou E), a partir dos escores alcançados. A letra A refere-se à classe social mais alta e a $\mathrm{E}$, a mais baixa.

As demais variáveis investigadas foram: sexo, estado civil, escolaridade, moradia, atividade laboral, tipo de atividade laboral, uso de álcool, fumo e café.

Para avaliar a presença de depressão foram utilizadas a Escala de Hamilton (HAM-D) ${ }^{(8)}$ e a entrevista clínica do DSM-IV. A HAM-D é um instrumento composto de 21 perguntas pontuadas quantitativamente: $10 \mathrm{com} 5$ alternativas (nunca, algumas vezes, bastante vezes, quase sempre e sempre) e 11 com 3 alternativas (nunca, algumas vezes e bastante vezes). A somatória dos pontos da escala originou uma variável contínua, posteriormente dicotomizada, tendo sido estabelecido o ponto de corte em mais de 17 pontos, que caracteriza depressão moderada ${ }^{(20)}$. A confiabilidade da escala varia entre 0,7 e 0,9 e a validade varia de 0,67 a $0,9^{(18)}$.

A entrevista clínica do DSM-IV classifica as queixas e as disfunções do paciente em categorias diagnósticas ${ }^{(2)}$. O método consiste em observar o comportamento do paciente e em motiválo a descrever seus problemas detalhadamente. O entrevistador traduz suas observações em termos de sinais e sintomas para um diagnóstico descritivo (Transtornos dos Eixos I e II), classificando-os em categorias nosológicas. Um diagnóstico dessa natureza, embora ajude a prever a evolução futura e a selecionar o tratamento mais eficaz, não possibilita conclusões quanto as suas causas.

Os pacientes que apresentaram sintomas sugestivos de depressão (acima de 17 pontos na HAM-D), foram posteriormente encaminhados para a entrevista clínica do DSM-IV para a confirmação do diagnóstico.Essa entrevista foi realizada por um único psiquiatra, integrante da equipe de pesquisa, que desconhecia a avaliação anterior do paciente.

Os dados foram inseridos por duas digitadoras, de forma independente, em banco de dados programado para amplitude e consistência no programa EpiInfo. A dupla entrada de dados visava identificar eventuais erros de digitação. Também foi realizada verificação automática dos dados no momento da digitação, através do Check do EpiInfo. 
A edição final dos dados e a análise foram realizadas no programa SPSS 10.1. Inicialmente procedeu-se à análise univariada para descrição das freqüências das variáveis de interesse. O teste do qui-quadrado foi utilizado na comparação entre proporções e a regressão logística não condicional, na análise multivariada Nessa análise a significância estatística referente à introdução de cada variável no modelo, foi avaliada através do teste de razão de verossimilhança. A técnica da regressão logística foi utilizada para estabelecer a chance de os pacientes com depressão apresentarem $\mathrm{DF}$, visto que a intenção era verificar os fatores a ela associados e controlar o efeito das variáveis intervenientes sobre a variável de desfecho. Dessa forma, os dados foram analisados hierarquicamente: no primeiro nível, incluíram-se as variáveis socioeconômicas, que no teste de razões de verossimilhança apresentaram valor de $P \leq 0,20$ e no segundo, a variável depressão. No modelo hierarquizado, as variáveis selecionadas no primeiro nível permaneceram no modelo subseqüente e foram consideradas como fatores associados à DF Cabe recordar que o desfecho DF nesta amostra representa o oposto do desfecho DO, uma vez que a amostra é constituída somente por pacientes dispépticos funcionais.

\section{RESULTADOS}

Dos 580 pacientes avaliados, 348 foram diagnosticados como dispépticos. Desses, 223 apresentavam dispepsia com causas orgânicas e 125 eram dispépticos funcionais (Figura 1). Inicialmente foi avaliada a presença de depressão nos grupos de DO e DF, constatando-se sua presença significativamente maior no grupo DF $(30,4 \%)$ em relação ao grupo DO $(11,2 \%)$ $(P<0,01)$ (Tabela 1$)$

TABELA 1 - Distribuição das variáveis sociodemográficas e depressão de acordo com o tipo de dispepsia (funcional ou orgânica) valor de $P$ da análise bivariada. Pelotas, RS, 2001

\begin{tabular}{|c|c|c|c|c|c|}
\hline \multirow[b]{2}{*}{ Variável } & \multicolumn{2}{|c|}{ Dispepsia funcional } & \multicolumn{2}{|c|}{ Dispepsia orgânica } & \multirow[b]{2}{*}{$P$} \\
\hline & $\mathrm{n}$ & $\%$ & $\mathrm{n}$ & $\%$ & \\
\hline \multicolumn{6}{|l|}{ Renda familiar (SM) } \\
\hline$\leq 2 \mathrm{SM}$ & 37 & 29,6 & 60 & 26,9 & \multirow{3}{*}{0,46} \\
\hline De 2 a 4 SM & 60 & 48,0 & 122 & 54,7 & \\
\hline$>5 \mathrm{SM}$ & 28 & 22,4 & 41 & 18,4 & \\
\hline \multicolumn{6}{|l|}{ Estado Civil } \\
\hline Casado & 72 & 57,6 & 136 & 60,9 & \multirow{3}{*}{0,38} \\
\hline Solteiro & 36 & 28,8 & 40 & 18,2 & \\
\hline Separado/Viúvo & 17 & 13,6 & 47 & 20,9 & \\
\hline \multicolumn{6}{|l|}{ Escolaridade } \\
\hline 0 a 4 anos & 32 & 26,0 & 56 & 25,1 & \multirow{3}{*}{0,86} \\
\hline 5 a 8 anos & 45 & 36,6 & 88 & 39,5 & \\
\hline$>8$ anos & 46 & 37,4 & 79 & 35,4 & \\
\hline \multicolumn{6}{|l|}{ Idade } \\
\hline Até 30 anos & 28 & 22,4 & 17 & 7,6 & \multirow{4}{*}{0,00} \\
\hline 31 a 50 anos & 60 & 48,0 & 161 & 72,2 & \\
\hline 51 a 60 anos & 22 & 17,6 & 38 & 17,0 & \\
\hline 61 ou mais & 15 & 12,0 & 7 & 3,1 & \\
\hline \multicolumn{6}{|l|}{ Gênero } \\
\hline Masculino & 35 & 28,0 & 92 & 41,8 & \multirow[t]{2}{*}{0,011} \\
\hline Feminino & 90 & 72,0 & 128 & 58,2 & \\
\hline \multicolumn{6}{|l|}{ Depressão } \\
\hline Presença & 38 & 30,4 & 25 & 11,2 & \multirow[t]{2}{*}{0,00} \\
\hline Ausência & 87 & 69,6 & 198 & 88,8 & \\
\hline
\end{tabular}

SM = salário mínimo
Contudo, foi necessário verificar se não houve diferenças significativas entre os grupos nas variáveis socioeconômicas que pudessem interferir nesse resultado. Nesse exame foram constatadas diferenças significativas entre os grupos nas variáveis idade e gênero (Tabela 1). Em relação à idade, existe grande concentração de pacientes com DO na faixa entre $31 \mathrm{e}$ 50 anos $(72,2 \%)$, enquanto entre os pacientes com DF, existe maior distribuição entre as faixas etárias $(22,4 \%$ até 30 anos, $48 \%$ entre 31 e 50 anos, $17,6 \%$ entre 51 e 60 anos e $12 \%$ com 61 anos ou mais) $(P<0,01)$. A distribuição dos pacientes em relação ao gênero foi a seguinte: entre os dispépticos funcionais $72 \%$ são mulheres, enquanto nos dispépticos orgânicos as pacientes do sexo feminino correspondem a $58,2 \%(P<0,001)$.

No segundo momento da análise estatística, a técnica de regressão logística foi realizada para identificar os fatores associados à DF e que a distinguem da DO (Tabela 2). Através dessa análise, verificou-se a associação entre DF e as variáveis estado civil e gênero: na primeira, os solteiros apresentaram 1,7 vezes mais chance de apresentar DF e na segunda, as mulheres que apresentaram chance 1,85 maior de desenvolver DF. Nessa análise, os pacientes com depressão apresentaram chance 3,46 vezes maior (IC 95\%, 1,15 a 2,97) de apresentar DF (Tabela 2). Contudo, as diferenças significativas apresentadas nos resultados das variáveis estado civil e gênero tornaram necessitaram uma análise multivariada complementar com o objetivo de controlar a influência destas variáveis intervenientes sobre a variável de desfecho, DF.

TABELA 2 - Prevalência de dispepsia funcional (razão de odds IC 95\%) e fatores sociodemográficos e depressão. Pelotas, RS, 2001

\begin{tabular}{|c|c|c|}
\hline Variáveis & $\mathrm{n}$ & Razão de odds (IC 95\%) \\
\hline \multicolumn{3}{|l|}{ Renda familiar (SM) } \\
\hline$\leq 2 \mathrm{SM}$ & 37 & $1,10(0,59-2,08)$ \\
\hline de 2 a 5 SM & 60 & $1,38(0,78-2,45)$ \\
\hline$>5 \mathrm{SM}$ & 28 & Referência \\
\hline \multicolumn{3}{|l|}{ Estado Civil } \\
\hline Casado & 72 & Referência \\
\hline Solteiro & 36 & $1,70(1,00-2,89)$ \\
\hline Separado/Viúvo & 17 & $0,68(0,36-1,27)$ \\
\hline \multicolumn{3}{|l|}{ Escolaridade } \\
\hline 0 a 4 anos & 32 & $0,98(0,56-1,73)$ \\
\hline 5 a 8 anos & 46 & $0,88(0,53-1,46)$ \\
\hline$>8$ anos & 47 & Referência \\
\hline \multicolumn{3}{|l|}{ Idade } \\
\hline Até 30 anos & 28 & Referência \\
\hline 31 a 50 anos & 60 & $0,23(0,12-0,44)$ \\
\hline 51 a 60 anos & 22 & $0,35(0,16-0,78)$ \\
\hline 61 ou mais & 15 & $1,30(0,44-3,83)$ \\
\hline \multicolumn{3}{|l|}{ Gênero } \\
\hline Masculino & 35 & Referência \\
\hline Feminino & 90 & $1,85(1,15-2,97)$ \\
\hline \multicolumn{3}{|l|}{ Depressão } \\
\hline Presente & 38 & $3,46(1,15-2,97)$ \\
\hline Ausente & 87 & Referência \\
\hline
\end{tabular}


A Figura 2 mostra os resultados da análise multivariada realizada através da técnica de regressão logística. Nesta análise, permaneceram associados à DF o gênero e a idade. As mulheres continuaram apresentando maior chance de DF (OR: 1,74, IC $95 \%, 1,05-2,89)$ e em relação á idade, os intervalos entre 31 e 50 anos (OR:0,28 IC 95\%, 0,13-0,54) e de 51 a 60 anos (OR: 0,41 , IC 95\%, 0,17-0,96) continuaram apresentando efeito protetor, ou seja, indivíduos nessas faixas etárias têm menor risco de apresentar DF.

\begin{tabular}{|c|c|c|}
\hline $\begin{array}{l}\text { Estado civil } \\
\text { Casado - Referência } \\
\text { Solteiro - 1,47 }(0,82 \text { - 2,64) } \\
\text { Separado/Viúvo - 0,50 (0,28 - 1,10) }\end{array}$ & $\begin{array}{l}\text { Sexo } \\
\text { Masculino - Referência } \\
\text { Feminino - 1,74 (1,05 - 2,89) }\end{array}$ & $\begin{array}{l}\text { Idade } \\
\text { Até } 30 \text { - Referência } \\
31 \text { a } 50-0,28(0,13-0,57) \\
51 \text { a } 60-0,41(0,17-0,96) \\
\text { Mais de } 60-1,80(0,57-5,62)\end{array}$ \\
\hline & $\begin{array}{l}\text { Depressão } \\
\text { Não - Referência } \\
\text { Sim - 3,10 (1,70 - 5,65) }\end{array}$ & \\
\hline
\end{tabular}

FIGURA 2 - Modelo hierárquico final para dispepsia funcional (IC 95\%)

A análise multivariada mostra também que, apesar dos fatores gênero e idade estarem associados, esses não anulam a associação entre depressão e DF. Os pacientes deprimidos têm três vezes mais chances de apresentarem depressão quando comparados aos dispépticos orgânicos (OR 3.10 IC 95\%, -1,71 -5,65). Após a análise multivariada descrita, a chance de deprimidos apresentarem DF continuou significativamente maior quando comparados com os não-deprimidos (OR: 3,10, IC 95\%,-1,71 -5,65).

Dessa forma, a associação entre DF e depressão foi confirmada por dois diferentes tipos de análise: a prevalência e os fatores associados.

\section{DISCUSSÃO}

Este estudo buscou verificar, através de um modelo de diagnóstico consistente, se a depressão é quadro mais prevalente entre pacientes com DF do que os com DO. O trabalho também procurou examinar a existência e a magnitude da associação entre DF e fatores socioeconômicos e entre depressão e DF.

É importante assinalar a utilização, no presente estudo, da entrevista clínica como um instrumento de confirmação do diagnóstico de depressão, fato incomum em estudos sobre o tema os quais, em sua maioria, utilizam apenas testagem, o que impossibilita a confirmação do diagnóstico. Além disso, buscou-se estabelecer as chances de pacientes com depressão apresentarem DF. Na revisão da literatura realizada pela equipe de pesquisa em bases de dados científicas consagradas (SciELO, Medline e PsicoInfo), não foram encontrados trabalhos sobre o tema no Brasil.

Os resultados evidenciaram maior prevalência $(P<0,01)$ de deprimidos entre os pacientes com DF $(30,4 \%)$ em relação àqueles com DO $(11,2 \%)$. Esses dados vão ao encontro dos achados de outros estudos de metodologia semelhante (avaliação da depressão através de escalas) ${ }^{(10,15,28)}$ em que também houve maior percentual de deprimidos entre pacientes com DF quando comparados aos pacientes com DO. A utilização da entrevista clínica para a confirmação de diagnóstico de depressão, procedimento utilizado no presente trabalho, foi encontrada em apenas outro estudo $^{(25)}$ que teve o diagnóstico de depressão confirmado pela entrevista clínica em $20,5 \%$ dos pacientes.

O passo seguinte do trabalho, uma análise da associação entre fatores socioeconômicos e DF, mostrou resultados entre as variáveis idade, sexo feminino e estado civil. Esse dado é semelhante ao de um estudo sobre prevalência de dispepsia na cidade de Pelotas ${ }^{(21)}$.

A análise muitivariada (que buscou controlar o efeito das variáveis citadas sobre o desfecho) confirmou a associação entre depressão e DF (OR: 3,10 IC 95\%, 1,71-5,65). Essa análise demonstrou que, na amostra estudada, os deprimidos têm mais chances de apresentarem DF. Em relação às características dessa amostra, deve-se assinalar que o presente estudo foi realizado somente na população que procurou atendimento médico e, portanto, não pode se inferir se a mesma associação deva ser encontrada nos pacientes com DF que não utilizaram os serviços de saúde. Contudo, em estudo de base populacional ${ }^{(14)}$ sobre transtornos gastrointestinais funcionais, utilizando o Critério de Roma I, foi encontrada associação entre DF e morbidade psicológica, sendo que neuroticismo, depressão, ansiedade e angústia somática mostraram-se preditores para DF. Por outro lado, estudo mais recente ${ }^{(11)}$ aponta para um percentual significativamente maior de deprimidos entre os pacientes com DF que procuraram atendimento médico, quando comparados àqueles que não o procuraram.

A co-morbidade entre depressão e DF tem despertado opiniões antagônicas sobre o significado e as causas dessa associação: os sintomas psicológicos seriam parte da causa da doença ou decorrem do sofrimento provocado pelos sintomas de dispepsia? Existe atividade de retroalimentação entre sintomas psíquicos e somáticos? O delineamento do presente trabalho não permitiu que fossem feitas inferências causais, podendo-se estar perante um viés de causalidade reversa. Entretanto, o mesmo propicia indicativo de que a presença de depressão deva ser levada em consideração durante avaliação do paciente e definição da estratégia de tratamento.

Uma limitação deste estudo é a não utilização de grupo comparativo oriundo da população geral. Contudo, em estudo de base populacional na mesma região ${ }^{(1)}$, foi encontrada prevalência de $10,2 \%$ de depressão (valor aproximado àquele encontrado entre os pacientes com DO $(11,2 \%)$ e inferior ao encontrado entre os pacientes com DF (30,4\%)).

Assim sendo, esses resultados permitem sugerir, como já o fazem outros estudos ${ }^{(10)}$, que um grupo de pacientes com DF poderia se beneficiar de programas de tratamento caracterizados por acompanhamento contínuo e suporte emocional. TALLEY ${ }^{(33)}$ oferece um guia para o manuseio da DF na clínica diária. Segundo esse autor, a utilização de terapia comportamental, psicoterapia, ou antidepressivos deve ser considerada se a erradicação do H. pilory, o tratamento baseado no sintoma predominante e/ou a terapia com inibidor da bomba de próton falharam em obter melhoras. Em relação ao uso de antidepressivo, uma meta-análise ${ }^{(13)}$ que examinou 12 
ensaios clínicos sobre transtornos gastrointestinais, buscou identificar os efeitos do uso dessa medicação. Seus resultados sugerem que, em especial os antidepressivos tricíclicos, parecem ser efetivos na melhora de um em cada três pacientes tratados. Entretanto, ainda segundo o artigo, se essa melhora é independente de tratamento farmacológico, é algo que carece de maior avaliação.

Dentro dessa linha de investigação, outra meta-análise sobre o efeito do tratamento da depressão sobre o esvaziamento gástrico ${ }^{(5)}$, indicou que a melhora clínica da depressão está relacionada a um esvaziamento gástrico mais eficiente, ao mesmo tempo em que afirmou que a melhora não poderia ser atribuída à ação farmacológica sobre o estômago. Por outro lado, estudo recente $^{(3)}$ indica que os mesmos antidepressivos tricíclicos foram efetivos apenas no tratamento de dor abdominal de pacientes com síndrome do intestino irritável, sem evidências que justifiquem sua recomendação na DF.

Assim, ainda é incerto se os inibidores seletivos da recaptação de serotonina (SSRIs) sejam alternativas de tratamento efetivas para $\mathrm{DF}$, pois parecem ser mais úteis em pacientes com constipação funcional (por suas qualidades procinéticas), embora isso ainda não tenha sido amplamente estudado. Ampliando a polêmica sobre o tema, outro estudo também recente ${ }^{(22)}$, mostrou efetividade da amitriptilina sobre os sintomas de DF. Para além da discussão sobre efetividade do tratamento, é importante que se considerem os efeitos colaterais dos psicofármacos (cólicas, náusea e diarréia no caso dos SSRIs pelo seu efeito procinético, e constipação, no caso dos antidepressivos tricíclicos).
Meta-análise sobre a utilização de psicoterapia na $\mathrm{DF}^{(26)}$ sugeriu a eficácia de tratamento psicológico, seja ele com um grupo de apoio ou através de terapia cognitivo-comportamental ou psicoterapia psicodinâmica interpessoal. Entretanto, os benefícios a longo prazo não foram comprovados. Mesmo assim o trabalho indica que a intervenção psicológica pode ser benéfica em casos selecionados de DF.

A produção de estudos sobre esse tema é complexa, já que tratamentos psicológicos são pouco utilizados como modalidade terapêutica para pacientes com distúrbios gastrointestinais funcionais. Em cuidados primários de saúde e em clínicas de gastroenterologia menos de $10 \%$ dos casos são tratados com psicoterapia ${ }^{(23)}$. Essa opção é, geralmente, considerada apenas para pacientes com sintomas psicológicos mais evidentes ou para aqueles cujo tratamento convencional não foi eficaz ${ }^{(30)}$. A definição da melhor estratégia de tratamento para os pacientes que mostram associação entre depressão e DF ainda não está estabelecida, assim como não estão claramente estruturadas quais medidas terapêuticas e de apoio social podem ser mais efetivas para uma melhora tanto no quadro depressivo, quanto na sintomatologia gastrointestinal.

Concluindo, os resultados mostram forte associação entre DF e depressão em um significativo grupo de pacientes e, ao mesmo tempo, sugerem a importância de uma definição de estratégias para o diagnóstico de depressão nesse mesmo grupo, bem como a definição de modelos específicos de tratamento.

Silva RA, Pinheiro RT, Silva RA, Horta BL, Moraes I, Faria AD. Functional dyspepsia and depression as an associated factor. Arq Gastroenterol. 2006;43(4):293-8.

ABSTRACT - Aim - To assess the association between depression and functional dyspepsia. Patients and methods - Three hundred and forty eight dyspeptic patients were included in a cross-sectional study in the gastroenterology outpatient clinic of a University Hospital in southern Brazil (Pelotas, RS) within a 1-year period (from March, 2001 to March, 2002). The assessment of depression occurred after the diagnosis of dyspepsia. The presence (or not) of depression was verified in both functional and organic dyspeptic patients. In a second moment, the results were compared, an univariate analysis was used to describe the frequencies of the interest variables and a chi-square for the comparison between proportions of the categorical variables. The logistic regression technique was used to establish the odd of functional dyspeptic patients to present depression and to control the effect of other variables in the outcome variable. Results - Showed greater prevalence of depression among functional dyspepsia patients (30,4\%), when compared to organic dyspepsia patients $(11,2 \%)$. Women showed greater risk to present functional dyspepsia (OR: 1,74, IC 95\%, 1,05-2,89) and in terms of age, the group with ages between 31 to 50 years (OR: 0,28 IC 95\%, 0,13-0,54) and 51 to 60 years (OR: 0,41, IC 95\%, 0,17-0,96) showed protection effect (subjects within this age groups have minor risk to present functional dyspepsia). After the multivariate analysis depressed patients showed three times greater comorbidity with functional dyspepsia when compared to non-depressed patients (OR 3, 13; IC 95\%; 1, 71-5, 74). Discussion - The adjustment for the gender, age and marital status variables confirmed the association between functional dyspepsia and depression. Results point the need to assess the presence of depression in functional dyspepsia patients and to establish specific treatment strategies for these patients.

HEADINGS - Depression. Dyspepsia. 


\section{REFERÊNCIAS BIBLIOGRÁFICAS}

1. Almeida-Filho N, Mari JJ, Coutinho E, Franca JF, Fernandes J, Andreoli SB, Busnello ED. Brazilian multicentric study of psychiatric morbidity - methodological features and prevalence estimates. Br J Psychiatry. 1997;171:524-9.

2. American Psychiatric Association. DSM-IV: manual diagnóstico e estatístico de transtornos mentais. 4" ed. Porto Alegre: Artes Médicas; 1995.

3. Bixquert-Jimenez M, Bixquert-Pla L. Tratamiento com antidepresivos en los transtornos funcionales digestivos Gastroenterol Hepatol. 2005;28:485-92.

4. Chou LT, Wu CY, Chen HP, Chang CS, Wong PG, Ko CW. The correlation of depression and gastric dysrhythmia in functional dyspepsia. J Clin Gastroenterol. 2001;33:127-31.

5. Demetrio FN, Soares MNT, Moreno RA, Costa PLA, Lopasso FP, Laudanna AA Efeito do tratamento da depressão sobre o esvaziamento gástrico. Rev Bras Psiquiatr. 1999;21:6-11.

6. Dotevall G. Functional dyspepsia: physiology, diagnosis and treatment. Stress Med 1994;10:101-5.

7. Drossman DA, Corazziari E, Talley NJ, Thompson G, Whitehead W, editors. Rome II - the functional gastrointestinal disorders. $2^{\text {nd }}$ ed. McLean, VA: Degnon Associates; c2000. p.299-350.

8. Hamilton M. Development of a rating scale for primary depressive illness. Br J Soc Clin Psychol. 1967;6:278-96.

9. Haug TT, Svebak S, Wilhelmsen I, Berstad A, Ursin H. Psychological factors and somatic symptoms in functional dyspepsia. A comparison with duodenal ulcer and healthy controls. J Psychosom Res. 1994;38:281-91.

10. Haug TT, Wilhelmsen I, Ursin H, Berstad A. What the real problems for patients with functional dyspepsia? Scand J Gastroenterol. 1995;30:97-100.

11. Haug TT. [Functional dyspepsia - a psychosomatic disease]. Tidsskr Nor Laegeforen 2002;122:1218-22

12. Herschbach P, Henrich G, Von Rad M. Psychological factors in functional gastrointestinal disorders: characteristics of the disorder or of the illness behavior? Psychosom Med 1999;61:148-53.

13. Jackson JL, O'Malley PG, Tomkins G, Balden E, Santoro J, Kroenke K. Treatment of functional gastrointestinal disorders with antidepressant medications: a meta-analysis. Am J Med. 2000;108:65-72.

14. Koloski N, Talley N, Boyce P. Epidemiology and health care seeking in the functional GI disorders: a population base study. Am J Gastroenterol. 2002;97:2290-9.

15. Langeluddecke $P$, Goulston $K$, Tennant C. Psychological factors in dyspepsia of unknown cause: a comparison with peptic ulcer disease. J Psychosom Res. 1990;34:215-22.

16. Lee S, Park MC, Choi SC, Nah Y, Abbey SE, Rodin G. Stress, coping, and depression in non-ulcer dyspepsia patients. J Psychosom Res. 2000;49:93-9.

17. Malte EA, Berle JE, Olafsson S, Lund A, Ursin H. Fibromyalgia is associated with panic disorder and functional dyspepsia with mood disorders. A study of women with random sample population controls. J Psychosom Res. 2000;49:285-9.

18. McDowell I, Newell C. Measuring health: a guide to rating scales and questionnaires. New York: Oxford University Press; 1996.
19. Mine K, Kanazawa F, Hosoi M, Kinukawa N, Kubo C. Treating nonulcer dyspepsia considering both functional disorders of the digestive system and psychiatric conditions. Dig Dis Sci. 1998;43:1241-7.

20. Moreno RA, Moreno DH. Escalas de avaliação clínica em psiquiatria e psicofarmacologia: escalas de avaliação para depressão de Hamilton (HAM-D) e Montgomery-Asberg (MADRS). Rev Psiq Clin (São Paulo). 1998;25:1-17.

21. Oliveira $\mathrm{S}$. Prevalência e fatores associados à dispepsia em adultos na cidade de Pelotas, RS, 1999-2000 [dissertação]. Pelotas, RS.: Universidade Federal de Pelotas; 2001.

22. Otaka M, Jin M, Odashina M, Matsuhashi T, Wada I, Horikawa Y, Ohba R, Oyake J, Hatakeyama N, Watanabe S. New strategies of therapy for functional dyspepsia using mosapride and amytriptilyne. Aliment Pharmacol Ther. 2005;21:42-6.

23. Palsson $\mathrm{O}$, Whitehead W. The growing case of hypnosis as adjunctive therapy for functional gastrointestinal disorders. Gastroenterology. 2002;123:2132-47.

24. Poitras M, Verrier P, So C, Pâquet S, Bouin M, Poitras P. Group counseling psychotherapy for patients with functional gastrointestinal disorders- development of new measures for symptom severity and quality of life. Dig Dis Sci. 2002;47:1297-307.

25. Porcelli P, De Carne M. Criterion-related validity of the diagnostic criteria for psychosomatic research for alexithymia in patients with functional gastrointestinal disorders. Psychother Psychosom. 2001;70:184-8.

26. Soo S, Moayyedi P, Deeks J, Delaney B, Lewis M, Forman D. Psychological interventions for non-ulcer dyspepsia. Cochrane Database Syst Rev. 2000;:CD001960.

27. Strid H, Norström M, Sjoberg J, Simrén M, Syedlund J, Abrahamsson H, Björnsson ES. Impact of sex and psychological factors on the water loading test in functional dyspepsia. Scand J Gastroenterol. 2001;36:725-30.

28. Talley NJ, Weaver AL, Zinsmeister AR, Melton LJ. Impact of functional dyspepsia on quality of life. Dig Dis Sci. 1995;40:584-9.

29. Talley NJ. Scope of the problem of functional digestive disorders. Eur J Surg. 1998;582:35-41.

30. Talley NJ, Silverstein M, Agréus L, Nyrén O, Sonnenberg A, Holtmann G. AGA technical review: evaluation of dyspepsia. American Gastroenterological Association. Gastroenterology. 1998;114:582-95.

31. Talley NJ, Stanguellini V, Heading RC, Koch KL, Malagelada JR, Tytgat GN. Functional gastroduodenal disorders. Gut. 1999;45 suppl 2:II37-42.

32. Talley NJ, Stanghellini V, Heading R. Functional gastroduodenal disorders. In: Drossman DA, Corazziari E, Talley NJ, Thompson G, Whitehead W, editors. Rome II - the functional gastrointestinal disorders. $2^{\text {nd }}$ ed. McLean, VA: Degnon Associates; c2000. p. $299-350$

33. Talley NJ. Dyspesia: management guidelines for the millenium. Gut. 2002;50:72-8.

34. Wiklund I, Glise H, Jerndal, Carisson J, Talley NJ. Does endoscopy have a positive impact on quality of life in dyspepsia? Gastrointest Endosc. 1998;47:449-54.

Recebido em 14/2/2005. Aprovado em 10/8/2006. 